\title{
Sensitivity, Specificity and Accuracy of Androgen Deficiency in Ageing Male (ADAM) Questionnaire for the Clinical Detection of Androgen Deficiency in the Male Population in Pakistan
}

\author{
Sidra Naz ${ }^{1}$, Nikeeta Mandhan ${ }^{2}$, Prem Shankar ${ }^{3}$, Kuldeep Raj ${ }^{4}$, Sidra Memon ${ }^{4}$ \\ 1. Internal Medicine, University of Health Sciences, Lahore, PAK 2. Internal Medicine, Dr. Ruth K. M. Pfau Civil \\ Hospital, Karachi, PAK 3. Internal Medicine, Dow University of Health Sciences, Karachi, PAK 4. Internal Medicine, \\ Jinnah Sindh Medical University, Karachi, PAK
}

Corresponding author: Sidra Memon, sidramemon311@gmail.com

\begin{abstract}
Introduction: Androgen deficiency in relation to the increasing age is quite prevalent worldwide. However, diagnosing it in low-income countries is quite a challenge due to cost concerns. Through this study, we plan to measure the sensitivity and specificity of the Androgen Deficiency in Ageing Male (ADAM) questionnaire in the Pakistani population.
\end{abstract}

Methods: A cross-sectional survey study was conducted from September 2019 to November 2019 in a Pakistani tertiary care hospital. Two hundred and fifty-five participants belonging to ages 30-69 years completed the ADAM Questionnaire in the out-patient department. Venous blood samples were taken to check serum total testosterone levels.

Results: The ADAM questionnaire revealed $90.12 \%$ sensitivity, $41.3 \%$ specificity, $45.34 \%$ positive predictive value, $90.80 \%$ negative predictive value, and $61.29 \%$ accuracy in the Pakistani population.

Conclusion: Low specificity and positive predictive value have been shown by the ADAM questionnaire. Hence, it cannot be used as a diagnostic tool to detect androgen deficiency, replacing the blood sample.

Review began 09/27/2020 Review ended 11/24/2020 Published 11/30/2020

(c) Copyright 2020

$\mathrm{Naz}$ et al. This is an open access article distributed under the terms of the Creative Commons Attribution License CC-BY 4.0., which permits unrestricted use, distribution, and reproduction in any medium, provided the original author and source are credited.
Categories: Endocrinology/Diabetes/Metabolism, Internal Medicine

Keywords: testosterone deficiency, pakistan, adam questionnaire

\section{Introduction}

Age-related androgen deficiency (AD) is a well-documented phenomenon in a range of studies. As a part of the normal ageing process, levels of testosterone fall around 0.5 to $2 \%$ per year starting from the fifth decade of life [1]. Deficiency of testosterone leads to a variety of symptoms including sexual dysfunction, psychological problems like depression and cognitive disability and physical symptoms like fatigue, osteoporosis and loss of body mass [2]. Testosterone deficiency can also predispose ageing males to atherosclerosis, leading to a number of cardiovascular diseases [3].

Serum testosterone level is the best confirmatory test to diagnose AD. Testosterone assays are not easily accessible in resource-poor countries like Pakistan, even though it is the gold standard. Henceforth, consistent efforts have been made to formulate questionnaires for screening of patients with $\mathrm{AD}$ [4-5]. Many questionnaires have been developed for screening patients with suspected androgen deficiency, however the most widely used questionnaire is the Saint Louis University Androgen Deficiency in Ageing Male (ADAM) questionnaire. It has three items: energy, mood and sexual function [4].

There is truly little research conducted on screening and diagnosis of androgen deficiency in Pakistan. The goal of this study is to measure the sensitivity and specificity of the Androgen Deficiency in Ageing Male (ADAM) questionnaire in the Pakistani population. It may help clinicians choose a screening tool for testosterone deficiency.

\section{Materials And Methods}

A cross-sectional survey was conducted from September 2019 to November 2019 in the Outpatient Department (OPD) of internal medicine in a tertiary care hospital in Pakistan. The study included 255 male participants age 30-69 years. These participants were suspected of testosterone deficiency based on their history and symptoms. Participants with recent illnesses, comorbidities such as renal disease, chronic liver disease and malignancies were excluded from the study, as these diseases may affect the measurement of total testosterone. Those who were on androgen therapy were also excluded. 


\section{Cureus}

The 10-item ADAM questionnaire was completed by the participants. The questions were asked by researchers themselves and each question was explained to participants. If the participants chose Yes to either one of the questions: decrease in libido or decrease in strength of erection or any other three questions, their ADAM test was positive [5]. Venous blood samples were collected for total testosterone levels. Liquid chromatography was used to measure total testosterone. To avoid any variation, the staples were collected between 10 am to $12 \mathrm{pm}$. As per American Urological Association, AD is defined as total testosterone less than $8 \mathrm{mmol} / \mathrm{l}$, which was our basis for the diagnosis [6].

Statistical Package for Social Sciences ${ }^{\circledR}$ software version 23.0 (SPSS; IBM Corp., Armonk, NY, USA) was used for data analysis. For numerical variables, data were expressed as means \pm standard deviations. Frequencies and percentages were used for categorical variables. Using analysis of variance (ANOVA), serum testosterone levels were checked for different age groups. Chi-square test was used to compare the ADAM test for different age groups. An online calculator (MedCalc, Ostend, Belgium) was used to calculate the sensitivity, specificity, positive predictive value, and negative predictive value for the overall ADAM questionnaire. A p-value less than 0.05 indicated that there is a difference in ADAM test and serum testosterone levels.

\section{Results}

The mean age of participants of $51 \pm 13$ years. A total of 168 participants had a positive ADAM test. The positive rate for the ADAM questionnaire increased significantly as the age increased (Table 1). The mean testosterone level $17.2 \pm 7.1 \mathrm{nmol} / \mathrm{l}$. The mean testosterone level significantly decreased with age (Table 2).

\begin{tabular}{|c|c|c|c|}
\hline \multirow{2}{*}{ Age } & \multicolumn{2}{|c|}{ Androgen Deficiency in Ageing Male (ADAM) questionnaire } & \multirow{2}{*}{ P-value } \\
\hline & Positive, n (\%) & Negative, n (\%) & \\
\hline $30-39(n=13)$ & $3(23.0 \%)$ & $10(77.0 \%)$ & \multirow{4}{*}{$0.0002^{*}$} \\
\hline $40-49(n=46)$ & $25(54.3 \%)$ & $21(45.6 \%)$ & \\
\hline $50-59(n=91)$ & 59 (64.8\%) & $32(35.2 \%)$ & \\
\hline $60-69(n=105)$ & $81(77.1 \%)$ & 24 (22.85\%) & \\
\hline
\end{tabular}

TABLE 1: Age versus ADAM Questionnaire Result

* means significant result

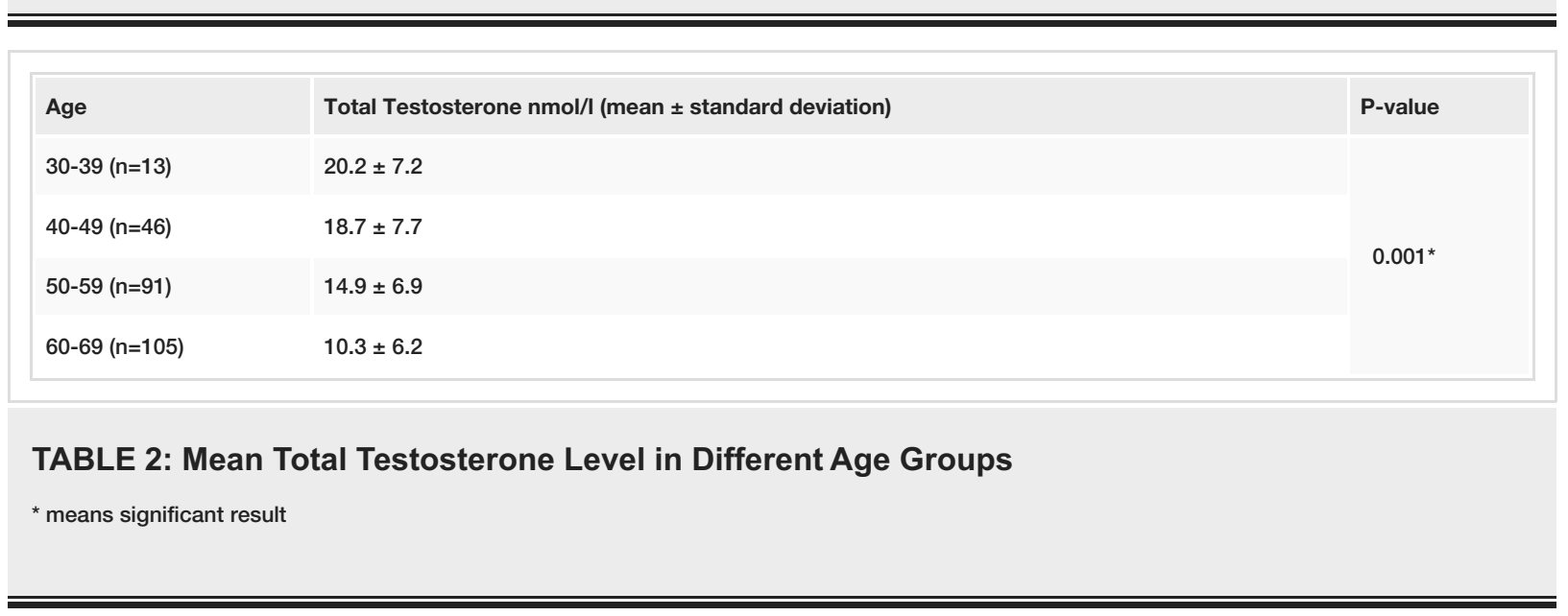

Our results indicated $90.12 \%$ sensitivity, $47.31 \%$ specificity, $45.34 \%$ positive predictive value (PPV), $90.80 \%$ negative predictive value (NPV) and 61.29\% accuracy of the ADAM questionnaire (Table 3). 


\section{Cureus}

\begin{tabular}{|c|c|c|c|c|c|c|c|}
\hline \multirow{2}{*}{$\begin{array}{l}\text { Androgen Deficiency in Ageing Male (ADAM) } \\
\text { questionnaire }\end{array}$} & \multicolumn{2}{|c|}{$\begin{array}{l}\text { Total } \\
\text { Testosterone }\end{array}$} & \multirow[t]{2}{*}{ Sensitivity } & \multirow[t]{2}{*}{ Specificity } & \multirow[t]{2}{*}{$\begin{array}{l}\text { Positive } \\
\text { Predictive Vale }\end{array}$} & \multirow[t]{2}{*}{$\begin{array}{l}\text { Negative } \\
\text { Predictive Value }\end{array}$} & \multirow[t]{2}{*}{ Accuracy } \\
\hline & Low & Normal & & & & & \\
\hline Positive & 73 & 88 & \multirow[t]{2}{*}{$90.12 \%$} & \multirow[t]{2}{*}{$47.31 \%$} & \multirow[t]{2}{*}{$45.34 \%$} & \multirow[t]{2}{*}{$90.80 \%$} & \multirow[t]{2}{*}{$61.29 \%$} \\
\hline Negative & 8 & 79 & & & & & \\
\hline
\end{tabular}

TABLE 3: Sensitivity and Specificity of the ADAM questionnaire

\section{Discussion}

Testosterone deficiency is common, such that $6 \%$ of adult males suffer from testosterone deficiency [7]. The incidence increases with increasing age and illness [7]. A meta-analysis concluded that decreased testosterone levels can lead to a $35 \%$ increased risk for mortality [8]. Without biochemical tests, testosterone deficiency is difficult to diagnose because of its vague symptoms [9].

In this research, the ADAM questionnaire exhibits $90.12 \%$ sensitivity, $47.31 \%$ specificity, $45.34 \%$ positive predictive value, $90.80 \%$ negative predictive value, and $61.29 \%$ accuracy. The ADAM questionnaire showed adequate sensitivity but as it lacks adequate specificity we can conclude that it is not a good alternative for serum testosterone assays. This was consistent with a study conducted in Kenya which showed an 88.1\% sensitivity and $44.7 \%$ specificity with $61.4 \%$ accuracy [10]. Chu et al. also found similar results in the Chinese population with $86 \%$ sensitivity and $40 \%$ specificity [11]. A similar study was conducted in the United States by Morley et al. reported $97 \%$ sensitivity and $30 \%$ specificity [12].

Ugwu et al. in their study discussed the reason for low specificity of the ADAM questionnaire. They mention that the ADAM questionnaire comprises three components; two of which (mood and energy) are susceptible to being affected by multiple factors and illnesses, other than androgen deficiency. This may be responsible for low specificity. They also determined the sensitivity and specificity for questions related to sexual functions. They found that specificity for the questionnaire for reduced libido is higher compared to the specificity of the ADAM questionnaire (75.5\% vs. 44.7\%). Based on this finding, Ugwu et al. recommended continuing using the ADAM questionnaire for screening, especially in low-income countries, as yes to the question related to loss of libido may be a good indicator for low testosterone levels [10].

As per our knowledge, this is the first study that has given the sensitivity, specificity and accuracy of the ADAM questionnaire for testosterone deficiency in Pakistan. However, there are certain limitations to this study. Total testosterone was used instead of bioavailable testosterone because of lack of resources. Also, all the samples were collected from a single center which narrows the results reserved to that population.

\section{Conclusions}

Low specificity, positive predictive value, and accuracy were found in this study. However, sensitivity and negative predictive values were satisfactory. We suggest that doctors should screen patients with the ADAM questionnaire since questions related to sexual function on the ADAM questionnaire might be helpful in the diagnosis of patients with testosterone deficiency. Considering there are limited resources, a more robust history should be taken before sending blood samples to determine total testosterone level. Further clinical studies should be conducted to make a questionnaire to screen patients with testosterone deficiency.

\section{Additional Information \\ Disclosures}

Human subjects: Consent was obtained by all participants in this study. Civil Hospital issued approval CHK/1904/11a. Animal subjects: All authors have confirmed that this study did not involve animal subjects or tissue. Conflicts of interest: In compliance with the ICMJE uniform disclosure form, all authors declare the following: Payment/services info: All authors have declared that no financial support was received from any organization for the submitted work. Financial relationships: All authors have declared that they have no financial relationships at present or within the previous three years with any organizations that might have an interest in the submitted work. Other relationships: All authors have declared that there are no other relationships or activities that could appear to have influenced the submitted work.

\section{References}

1. Harman SM, Metter EJ, Tobin JD, Pearson J, Blackman MR: Longitudinal effects of aging on serum total and free testosterone levels in healthy men. J Clin Endocrinal Metab. 2001, 86:724-31. 10.1210/jcem.86.2.7219

2. Rajfer J: Decreased testosterone in the aging male. Rev Urol. 2003, 5:S1-S2. 
3. Hak AE, Witteman JC, de Jong FH, Geerlings MI, Hofman A, Pols HA: Low levels of endogenous androgens increase the risk of atherosclerosis in elderly men: the Rotterdam Study. J Clin Endocrinal Metab. 2002, 87:3632-9. 10.1210/jcem.87.8.8762

4. Morley JE, Charlton E, Patrick P, Kaiser FE, Cadeau P, McCready D, Perry HM: Validation of a screening questionnaire for androgen deficiency in aging males. Metabolism. 2000, 49:1239-42.

10.1053/meta.2000.8625

5. Smith KW, Feldman HA, McKinlay JB: Construction and field validation of a self-administered screener for testosterone deficiency (hypogonadism) in ageing men. Clin Endocrinol (Oxf). 2000, 53:703-11.

6. Mulhall JP, Trost LW, Brannigan RE, et al.: Evaluation and Management of Testosterone Deficiency . American Urological Association, 2018.

7. McBride JA, Carson CC, Coward RM: Diagnosis and management of testosterone deficiency. Asian J Androl. 2015, 17:177-186. 10.4103/1008-682X.143317

8. Araujo AB, Dixon JM, Suarez EA, Murad MH, Guey LT, Wittert GA: Endogenous testosterone and mortality in men: a systematic review and meta-analysis. J Clin Endocrinol Metab. 2011, 96:3007-3019. 10.1210/jc.2011-1137

9. Vermeulen A, Kaufman JM: Ageing of the hypothalamo-pituitary-testicular axis in men. Horm Res. 1995, 43:25-8. 10.1159/000184233

10. Ugwu TE, Rosemary TI: Performance of the Androgen Deficiency in Aging Male questionnaire for the clinical detection of androgen deficiency in black sub-Saharan African men with Type-2 diabetes mellitus. J Endocrinol Metab Diabetes S Africa. 2017, 22:1-4. 10.1080/16089677.2017.1318534

11. Chu LW, Tam S, Kung AW, et al.: A short version of the ADAM questionnaire for androgen deficiency in Chinese men. J Gerontol A Biol Sci Med Sci. 2008, 63:426-31. 10.1093/gerona/63.4.426

12. Morley JE, Perry HM, Kevorkian RT, Patrick P: Comparison of screening questionnaires for the diagnosis of hypogonadism. Maturitas. 2006, 53:424-9. 10.1016/j.maturitas.2005.07.004 